Stephane H Maes, (2020), "Gravity or Magnetic Monopoles? You Cannot Have Both!“, viXra:2006.0190v1, or https://shmaesphysics.wordpress.com/2020/06/15/gravity-or-magnetic-monopoles-you-cannot-haveboth/, June 15, 2020.

\title{
Gravity or Magnetic Monopoles? You Cannot Have Both!
}

\author{
Stephane H. Maes ${ }^{1}$
}

June 15, 2020

\begin{abstract}
:
In this paper, we argue that gravity breaks the symmetry between electric and magnetic equations (without sources) within the Standard Model. As a result, the motivation for introducing magnetic monopoles disappears (without affecting charge quantization): magnetic monopole probably do not exist in the presence or gravity. It is consistent with the absence of observation of magnetic monopoles and resolves the magnetic monopole cosmology problem. This result could have serious implications for supersymmetry, supergravity, GUTs and ToEs, including in particular superstrings. Some of these implications, and possible ways forward, are discussed.
\end{abstract}

The new preprint [1] proposes contributions to several open problems in physics like the reconciliation of General Relativity with Quantum Physics, explaining the origin of gravity proposed as emerging from quantum (EPR-

Einstein Podolsky Rosen) entanglement between particles, detailing contributions to dark matter and dark energy and explaining other Standard Model mysteries without requiring New Physics beyond the Standard Model other than the addition of gravity to the Standard Model Lagrangian. All this is achieved in s multi-fold universe that may well model our real universe, which remains to be validated.

With the proposed model of [1], spacetime and Physics are modeled from Planck scales to quantum and macroscopic scales and semi classical approaches appear valid till very small scales. In [1], it is argued that spacetime is discrete, with a random walk-based fractal structure, fractional and noncommutative at, and above Planck scales (with a 2-D behavior and Lorentz invariance preserved by random walks till the early moments of the universe). Spacetime results from past random walks of particles. Spacetime locations and particles can be modeled as microscopic blackholes (Schwarzschild for photons and spacetime coordinates, and metrics between Reisner Nordstrom [19] and Kerr Newman [20] for massive and possibly charged particles - the latter being possibly extremal). Although surprising, [1] recovers results consistent with other like [21], while also being able to justify the initial assumptions of black holes from the gravity or entanglement model. The resulting gravity model recovers General Relativity at larger scale, as a 4-D process, with massless gravity, but also with massive gravity components at very small scale that make gravity significant these scales. Semi-classical models also work well till way smaller scales that usually expected.

It means that following [1], gravity can be added to the Standard Model Lagrangian [2] by adding gravity and entanglement terms.

Magnetic monopoles have been predicted for a long time (as far as Dirac), as an hypothetical magnetic charge, symmetrizing the Maxwell equations with sources. In particular, Dirac showed that the presence of magnetic monopoles, implies quantization of the electric charge as we observe in nature: all observed charges are in integer multiples of $+e$ or -e. This classical derivation can also be treated as a gauge quantum Field property [3]. History and details can be found in [4].

The quarks and the discovery of quasi particles in quantum material phenomena [5] threw some curved balls, by introducing fractional charges (in $\pm \frac{e}{3}$ ). Of course, one can then simply argue that the fundamental charge was $\frac{e}{3}$

\footnotetext{
${ }^{1}$ shmaes.physics@gmail.com
} 
instead of e, although hierarchical states create new problems with the quantum Hall effect as it can now be in $\frac{e}{m}$, where $\mathrm{m}$ are odd integers. Interestingly, as a result quasi magnetic monopoles analogues, i.e. not really Dirac's monopoles as elementary particles, were also observed (see references in [4]). Depending on the point of view, these magnetic monopoles analogues can be viewed as a confirmation of the mathematical model linking fractional charges and magnetic monopoles.

The quarks were also rigorously handled within the framework of Yang Mills Gauge Theory with the right symmetry groups, at small scales and within QCD confinement, and therefore confinement of these fractional charges [6]. Therefore, there are no issues with classical physics or quantum mechanics: everything is in integer multiples of +e o -e.

Today all Grand Unification Theories (GUTs)[8], and other supersymmetric models [9], and hence superstrings, as well as many Theories of Everything (ToEs)[10], all imply magnetic monopoles. This is always the case, at the difference of proton decay $[1,11]$, which is also often a consequence of these theories, but which is not always implied. Indeed, 't Hooft and Polyakov showed that magnetic monopole is unavoidable, whenever the $\mathrm{U}(1)$ gauge group of electrodynamics is embedded in a larger unified gauge group (e.g. see [7] for a more approachable review and more references). For example, All GUTs predict magnetic monopoles.

The problem is that no magnetic monopole was ever observed (except, maybe, for a few unconfirmed and never repeated strange events, a while ago, that probably were not monopoles [4]).

Yet, their existence are not really doubted.

[1] argues that magnetic monopoles probably do not exist! Two arguments are presented but they all amount to the impact of gravity. This would explain the lack of observations despite very significant efforts and create big challenges for all the theories predicting them $[4,13,14]$.

The idea is simple, with its gravity models, [1] is confident that in a multi-fold universe, gravity]s (and entanglement's) effective potentials can be added to the Standard Model Lagrangian and that semi-classical models also extend to way smaller cases that usually expected. In addition, with the massive gravity components that [1] predicts at very small scales, the effects are expected to be non-negligible.

Said differently, at the scales where GUTs play a role, gravity can have a large enough effect. And gravity destroys the symmetry between electric and magnetic fields [15] that is behind adding magnetic charges. Another way to look at it is that, as discussed in [1], particles appear associated to a uncertainty region, according to the uncertainty principle (and the hard no supra luminosity limit). It is not a point particle, but rather a ball of uncertainty. Within and around that region. Spacetime reconstruction models shows that spacetime, discrete as already mentioned, and particles are presented by microscopic black holes (possibly extremal for charged particles). The particle blackholes result from the structure of the effective potential surrounding every particle as a result of the EPR entanglement of all the virtual particles that are emitted by the particle. Spacetime points are rather the result of the spacetime concretization by passage of particles following random walks. Massless carriers like photons are also Schwarzschild blackholes. All these are relevant only at very small scales, below the scales of typical quantum models. This model describes spacetime and gravitation as 2-D processes at very small scales and 4-D at larger scales. The spacetime is Lorentz invariant, thanks to the random walk-based spacetime reconstruction that generates a fractal structure and a non-commutative geometry. GR equation can be recovered as a result of the model of [1] (by computation of path integrals, or by Thermodynamics arguments). The consequences of gravity (and curved spacetime) are that a charge appears as a current (and some would argue also as a string), with preferred directions (defined by the geodesics, without them, i.e. when there is no gravity and no geodesic (they are isotropic), uncertainties just increase the ball of the charge density and no symmetry breaking occurs but in their presence, currents appear for all charges), while a current remains as a current, possibly deformed: symmetry is broken for Maxwell equations with sources and between magnetic and electric fields. As a result polarity 
(helicity or spin) is no more conserved [15], flips occur, just as for massive and massless fermions [1], and the arguments for symmetrizing the equations, by adding a magnetic charge density, disappear.

These two arguments ([15] and the intuitive explanation above from [1]) argue against the symmetries behind the magnetic monopole. They are broken by gravity. Note that it does not necessarily affect charge quantization the same way: it could be argued to result from the approximate symmetries that still exists when in a flat space/ without gravity, à la [6], as charges are conserved and should not be affected by the presence or absence of gravity (think about the local gauge symmetry and charge conservation as preserved).

This conclusion could explain the lack of observation of the monopoles $[4,13,14]$ and resolve the magnetic monopole cosmology problem without requiring inflation [12] for example. Indeed, for the latter, gravity was present (and strong) when cosmological monopoles are conventionally thought to have formed. Our argument is that they never formed, because of gravity that never allowed the required symmetries to reign!

Yet the conclusions from [1], and this paper so far, along with the proton decay problem [1,11], are real problems with GUTs, ToEs, supersymmetry and superstrings [12]. Their options go as follow:

- GUTs are in big trouble. If [1] is right, then they are not valid, unless if they can drop the prediction of magnetic monopole (and proton decay) because of gravity (It may be ok as gravity is not included in their model).

- It may be a way out

- Yet it may not work for theories that are fundamentally built on a symmetry that can never reign (versus being broken later or at higher scales). Supersymmetry and GUTs do require supersymmetry to reign before being broken (in time or scale). Gravity prevents this from ever happening. What does that do to the model?

- Some ToEs that model gravity and predict magnetic monopoles are potentially in even bigger trouble, with little way out as they are supposedly already modeling gravity and hence such effects. This directly affects superstrings, M-theory, and SUGRA, supersymmetry with super gravity:

- ToEs that just encompass GUT and gravity could use the argument above with the challenges that we discussed in the previous main bullet

- All the others are supposed to already model gravity! So they supposed to model this symmetry breaking and not predict magnetic monopoles. If they do, there is a problem with the model.

- They either need to be able to eliminate that predict,

- or explain why our argument does not affect their prediction (or fault our reasoning of course).

For superstrings, we recommend considering the analysis presented in [1] and explore recommendations at the end of [16]:

- Evolve to positioning superstring as suggested in multi-fold universe [1]

- And / or consider the proposal that spacetime is outside the space of superstrings and have gravitons and other super partners live in $\operatorname{AdS}(5)$ (plus other dimensions), but no physical particles living there [16].

GUTs, supersymmetry with supergravity may also try to follow the second bullet (with maybe additional considerations like limiting super partners and magnetic monopoles to small (compact) dimensions with only virtual particle effects elsewhere and of course also dealing with the proton decay problem $[11,16])$; but we have not thought of all the implications or what it would really look like. It is for future works.

We believe that the implications of the work presented here are significant. The analysis does not solely rely on [1], in fact [15] would be enough. But [1] provides a framework where we can more confidently make our arguments about the impact and validity of gravity in a multi-fold universe. Yes we still have to validate or falsify if such a universe can model the real universe. In a few papers now $[1,11,16]$, we have presented arguments to that effect (see also [17] for related arguments in trying to prove other properties relevant to our universe). Without [1], and outside a multi-fold universe, the arguments presented here remain valid if gravity is strong enough at 
small scale to impact the standard model (and to do it as argued). We believe that this is a way lower bar, that many more people can more comfortably accept.

We realize that impact of this analysis, if correct, is very strong for Today's Physics. Hopefully, it will be seriously considered. Of course, weaknesses or mistakes in our arguments may leave other doors open to existing theories. Discovering magnetic monopole, despite the analysis of the present paper, would close the debate and prove us wrong, yet it would not negatively impact the proposal for multi-fold universe as magnetic monopoles are just a side consideration.

It is notable that much more attention has been paid to the lack of observation of the proton decay, despite also the lack of observation of magnetic monopoles. The fact that they are not associated to half-life estimates does give a firm energy range expected for observation of magnetic monopoles. That is probably why hopes are much higher that it is just a matter of time. Well, the present papers should ratchet the concerns a bit up.

Finally, it is important to know that there is another loophole for the discovery of magnetic monopoles: small magnetic black holes (i.e. remnants with magnetic flux). See [18], for suggestions that this may happen (Unfortunately, without enough references or background). Although [1] models particles and spacetime locations with microscopic blackholes, we do not believe that in this case the magnetic remnants would amount to particle (i.e. a magnetic monopole) per all the discussion above. Instead, they could just be remnant small magnetic black holes. So, if something is observed, it would be good to understand if they are magnetic monopole particles, and therefore prove us wrong and save all the theories that predict them, or magnetic remnants of no help for anybody, in this discussion, other than the progress of science.

References: (most references come from popular science to make the discussion more approachable)

[1]: Stephane H. Maes, (2020) “Quantum Gravity Emergence from Entanglement in a Multi-Fold

Universe", viXra:2006.0088v1, (June 9, 2020).

[2]: https://en.wikipedia.org/wiki/Mathematical formulation of the Standard Model

[3]: Yang C.N. (1996), "Magnetic Monopoles, Fiber Bundles, and Gauge Fields". In: Newman H.B., Ypsilantis T. (eds)

"History of Original Ideas and Basic Discoveries in Particle Physics". NATO ASI Series (Series B: Physics), vol 352.

Springer, Boston, MA

[4]: https://en.wikipedia.org/wiki/Magnetic monopole

[5]: https://en.wikipedia.org/wiki/Fractional quantum Hall effect

[6]: John Preskill, (1984), "Magnetic Monopoles", Ann. Rev. Nucl. Part. Sci., 1984. 34:461-530

[7]: Arttu Rajantie, (2012), "Magnetic monopoles in field theory and cosmology ", Phil. Trans. R. Soc. A.37057055717

[8]: https://en.wikipedia.org/wiki/Grand_Unified Theory

[9]: https://en.wikipedia.org/wiki/Supersymmetry

[10]: https://en.wikipedia.org/wiki/Theory of everything

[11]: Stephane H Maes, (2020), "Gravity Induced Anomalies Smearing in Standard Model so that Protons May

Never Decay, Except in Black

Holes", viXra:2006.0128v1, https://shmaesphysics.wordpress.com/2020/06/12/protons-may-never-decay-exceptin-black-holes/, June 13, 2020.

[12]: https://en.wikipedia.org/wiki/Inflation_(cosmology)

[13]: https://atlas.cern/updates/physics-briefing/new-result-magnetic-monopoles

[14]: http://physicsdetective.com/magnetic-monopoles/

[15]: Ivan Agullo, Adrian del Rio, and Jose Navarro-Salas, (2017), "Electromagnetic Duality Anomaly in Curved Spacetimes", Phys. Rev. Lett. 118, 111301

[16]: Stephane H Maes, (2020), " Dualities or Analogies between Superstrings and Multi-fold Universes", viXra:2006.0155v1, https://shmaesphysics.wordpress.com/2020/06/14/dualities-or-analogies-betweensuperstrings-and-multi-fold-universes/, June 14, 2020. 
[17]: Stephane H Maes, (2020), " Progresses on Proving the Mass Gap for Yang Mills and Gravity (maybe it's already proven...)", viXra:2006.0178v1, https://shmaesphysics.wordpress.com/2020/06/14/dualities-or-analogiesbetween-superstrings-and-multi-fold-universes/, June 14, 2020.

[18]: https://www.npl.washington.edu/AV/altvw01.html

[19]: https://en.wikipedia.org/wiki/Reissner\%E2\%80\%93Nordstr\%C3\%B6m metric

[20]: https://en.wikipedia.org/wiki/Kerr-Newman metric

[21]: Burinskii, Alexander, (2008), "The Dirac-Kerr-Newman electron", arXiv:0507109v4 